\title{
EL2 deep-level transient study in semi-insulating GaAs using positron-lifetime spectroscopy
}

\author{
Y. Y. Shan, C. C. Ling, A. H. Deng, B. K. Panda, C. D. Beling, and S. Fung \\ Department of Physics, University of Hong Kong, Pokfulam Road, Hong Kong
}

(Received 8 December 1995; revised manuscript received 29 May 1996)

\begin{abstract}
Positron lifetime measurements performed on $\mathrm{Au} / \mathrm{GaAs}$ samples at room temperature with an applied square-wave ac bias show a frequency dependent interface related lifetime intensity that peaks around $0.4 \mathrm{~Hz}$. The observation is explained by the ionization of the deep-donor level $E L 2$ to $E L 2^{+}$in the GaAs region adjacent to the $\mathrm{Au} / \mathrm{GaAs}$ interface, causing a transient electric field to be experienced by positrons drifting towards the interface. Without resorting to temperature scanning or any Arrhenius plot the EL2 donor level is found to be located $0.80 \pm 0.01 \pm 0.05 \mathrm{eV}$ below the conduction-band minimum, where the first error estimate is statistical and the second systematic. The result suggests positron annihilation may, in some instances, act as an alternative to capacitance transient spectroscopies in characterizing deep levels in both semiconductors and semi-insulators. [S0163-1829(97)01012-6]
\end{abstract}

\section{INTRODUCTION}

Positron annihilation lifetime (PAL) spectroscopy has proved to be a valuable nondestructive probe for vacancytype defects in GaAs, having revealed the presence of $V_{\mathrm{As}},{ }^{1,2}$ $V_{\mathrm{Ga}}{ }^{2,3}$ and vacancies associated with the metastable state of the native defect EL2 (Ref. 4) and the reverse contrast center. $^{3}$ Recently, however, another way of employing the positron to study defects in semiconductors has been suggested through measurements made of the positron mobility in GaAs which revealed the presence of the EL2 deep-donor level. ${ }^{5}$ In this alternative approach the positron essentially acts as an electric-field sensor within a depletion region monitoring defect charge state transients due to carrier emission in much the same way as conventional deep-level transient spectroscopy (DLTS) monitors these transients through junction capacitance. ${ }^{6}$ Indeed, since the reverse bias is the product of $E_{m}$, the mean electric field within a depletion region, and depletion width $d$, the junction capacitance $C$ and $E_{m}$ are directly related.

The possibility of using the positron as an electric-field probe arises through the intrinsic mobility of the particle in the semiconductor lattice, the positron being a light quantum particle with similar transport properties to the hole and electron. The particle's velocity being directly dependent on the electric field, $E$, allows variations in $E$ to be monitored by one of two methods. The first is that of observing the positron drift velocity through the Doppler shift it produces in the energy of the annihilation line. ${ }^{5}$ The second, which is the subject of this report, is less direct and relies upon observing the fraction of positrons that, under the action of the electric field, drift to an interface within the annihilation time of the particle. $^{7}$

The present work is a continuation of the PAL study performed previously in our laboratory in which significant fractions $\sim 10 \%$ of positrons implanted from a ${ }^{22} \mathrm{Na}$ radioactive source were electric field drifted into microvoids (with characteristic positron lifetime $\sim 400 \mathrm{ps}$ ) at the $\mathrm{Al} / \mathrm{GaAs}$ and $\mathrm{Au} / \mathrm{GaAs}$ interfaces upon application of a reverse bias. ${ }^{8}$ In this previous work (see Sec. IV of Ref. 8) an ac square wave was applied to the positron injecting contacts of the sand- wiched sample assembly with the reverse bias alternating between zero and $40 \mathrm{~V}$. In the reverse biased half cycle the $E L 2$ traps ionized to $E L 2^{+}$, causing the time-averaged interface microvoid intensity, $I_{2}$, to increase as the intensifying electric field drifted more positrons to the interface state. On the other hand, for zero bias, electrons diffusing into the space-charge region from the bulk, neutralized the EL2 traps, and reduced $I_{2}$ close to its dc zero-biased value. With increasing frequency the time available for the $E L 2^{+}$formation on the reverse-biased half cycle diminished, causing a drop in $I_{2}$ at frequencies consistent with the known electron emission rate the $E L 2$ donor exhibits at room temperature. A deficiency, however, with this preliminary investigation was that it led to no exact determination of the energy of the deep donor, preventing a definitive assignment with the EL2 defect. This was partly a result of no model being available to describe the charge transient effects on the positron drift, and partly a result of the incomplete donor neutralization on the trap filling half cycle. The motivation behind the present work was to address both of these aspects by performing further experiments over a more extensive frequency range.

\section{EXPERIMENT}

The samples used in the present study were undoped LEC grown semi-insulating (SI) $\mathrm{GaAs}(100)$ obtained from ICI Wafer Technology Ltd. with a room temperature resistivity of $10^{8} \Omega \mathrm{cm}$. The quoted free-carrier, $E L 2$ and $C$ concentrations were $6 \times 10^{7}, 1.5 \times 10^{16}$, and $10^{15} \mathrm{~cm}^{-3}$, respectively. The sample preparation, sample biasing and the lifetime spectrometer were identical to those used in our previous study and details may be found in Ref. 8. To reduce errors associated with source annihilations, however, the $\mathrm{Ni}$ foil encapsulated source was replaced by $5 \mu \mathrm{Ci}$ of ${ }^{22} \mathrm{NaCl}$ directly deposited onto one of the Au metalizations, the latter being earthed by way of thin $\mathrm{Au}$ wires attached by silver paint. An important improvement over the previous work was that instead of just removing the bias in the trap filling half of the cycle, the positron injecting contacts were subject to a $115 \mathrm{~V}$ forward bias so as to allow thermal carriers to penetrate more effectively the space-charge region for neu- 


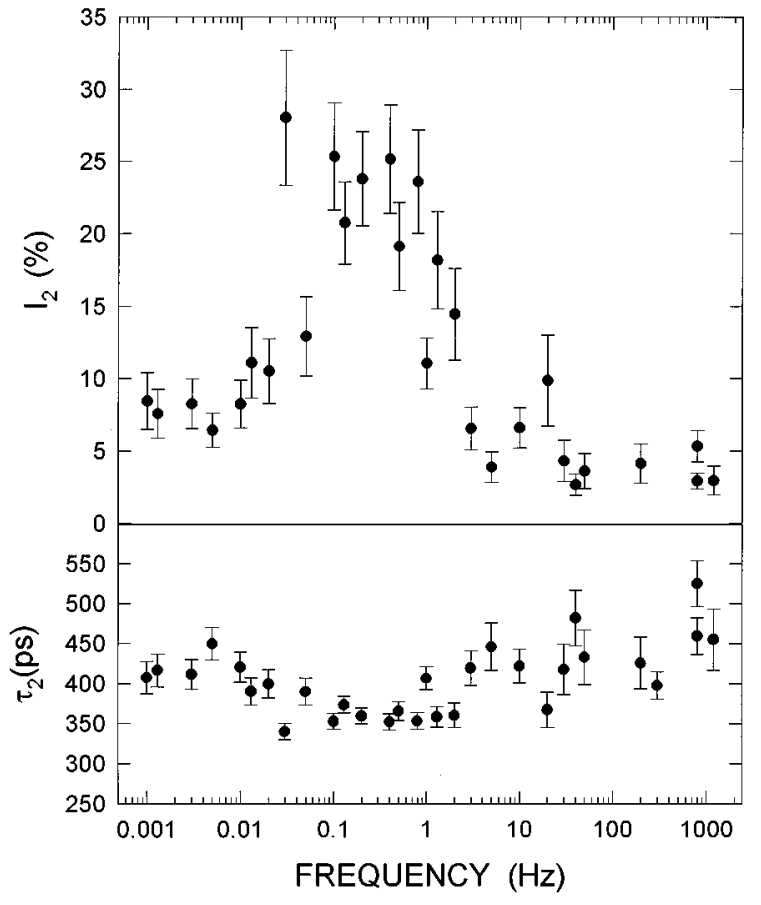

FIG. 1. The intensity $I_{2}^{r}$ and the lifetime $\tau_{2}$ of the long-lived interfacial (microvoid) spectral component in the positron lifetime spectra from $\mathrm{Au} / \mathrm{GaAs}$ SI samples on the $115-\mathrm{V}$ reverse-biased half cycle of an applied square-wave bias shown plotted against the bias frequency.

tralization of the ionized deep donors $\left(E L 2^{+}\right)$. This neutralization (or trap filling) process was not complete in the earlier study except at the lowest frequencies making theoretical modeling of the data difficult. The present study was performed at $295 \mathrm{~K}$, with $10^{6}$ counts collected per spectrum, over the more extended range of frequencies from $1 \mathrm{mHz}$ to $1.2 \mathrm{kHz}$. A synchronized gating logic pulse from the pulse generator allowed lifetime spectra to be collected in coincidence with either the reverse-biased or the forward-biased half cycles.

Following the procedure of our previous PAL study,${ }^{8}$ lifetime spectra were initially analyzed assuming only two exponential components. However, as a result of systematic changes in the lifetime $\tau_{2}$ and intensity $I_{2}$ of the fitted long component as spectra were collected with sequentially higher statistics of up to $8 \times 10^{6}$ counts, it was discovered that a low intensity $(0.05 \%) 1800$-ps component was also present in the spectra. This is most likely attributed to $o$-Ps associated with surfaces or spaces between source foils and sample. Both the intensity and lifetime of this third component were kept fixed in all subsequent spectral analyses to remove any perturbation to the important second component $(\sim 400 \mathrm{ps})$ associated with the $\mathrm{Au} / \mathrm{GaAs}$ interface. The resulting free-fitted $I_{2}$ and $\tau_{2}$ values are shown in Fig. 1 for spectra taken under the reverse-biased half cycle. As found in Ref. 8 the value of $I_{2}$ (reverse) falls significantly in the range of $1-100 \mathrm{~Hz}$ as a result of there being progressively less time for the $E L 2^{0} \rightarrow E L 2^{+}$transition. Also seen in Fig. 1 is a noticeable drop in $I_{2}$ as the frequency is reduced from $1 \mathrm{~Hz}$ to $1 \mathrm{mHz}$ (where the dc value for $I_{2} \sim 8 \%$ is obtained).

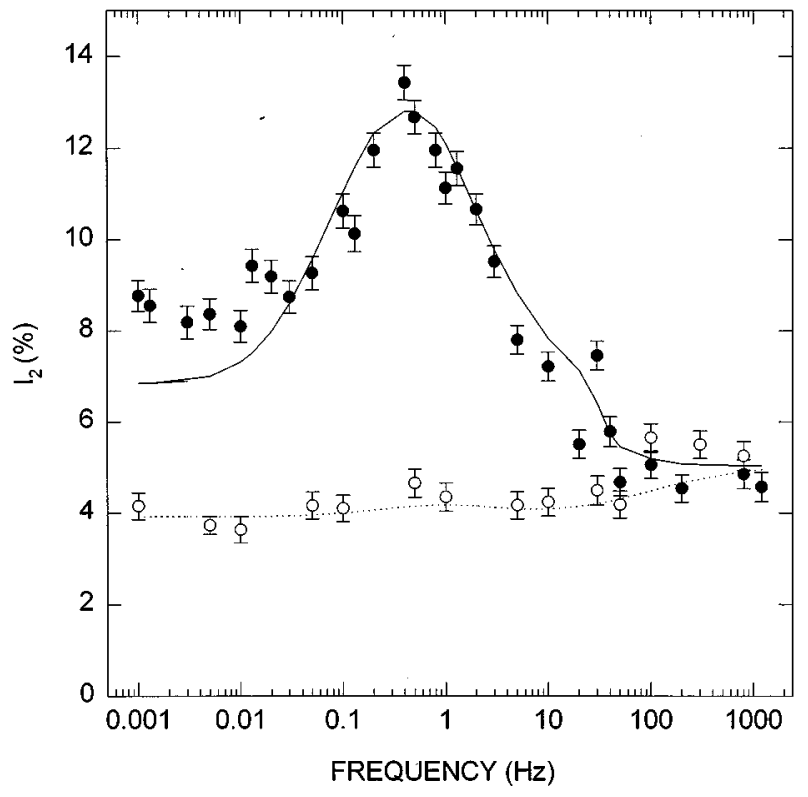

FIG. 2. The fitted $\mathrm{Au} / \mathrm{GaAs}(\mathrm{SI})$ interfacial intensities $I_{2}^{r}$ (solid circles) and $I_{2}^{f}$ (open circles) in the reverse-biased and forwardbiased half cycles, respectively, of the $\pm 115 \mathrm{~V}$ square-wave bias, plotted as a function of bias frequency. These data have been obtained by fixing $\tau_{2}$ at $410 \mathrm{ps}$. The solid and dotted lines show the fits to the $I_{2}^{\prime}$ and $I_{2}^{f}$ data using the deep-level transient model described in the text.

The $\tau_{2}$ values (see Fig. 1) show a scatter around $\sim 400 \mathrm{ps}$ in agreement with our previous study. ${ }^{8}$ However, it may be noted that the $\tau_{2}$ data exhibit a definite inverse correlation with $I_{2}$. This correlation is simply an artifact of least-squares fitting spectra with limited statistics $\left(10^{6}\right.$ counts $)$ and insufficient instrumental resolution. There are two undesirable features about this effect. The first is that it is difficult to ascertain the exact value of $\tau_{2}$ that is required for both correct extraction of $I_{2}$ and for modeling. The second is that a larger than expected scatter results in the fitted values of both $I_{2}$ and $\tau_{2}$. Reverse biased spectra collected with sequentially higher statistics, however, showed a convergence of $\tau_{2}$ to $410 \pm 4 \mathrm{ps}$ for coincidence counts above $6 \times 10^{6}$. This value was thus taken as being close to the real value of $\tau_{2}$ and was fixed in a subsequent fitting so as to remove the unwanted correlation effect and the associated amplified statistical scatter. The fitted results of $I_{2}$ in both reverse bias, $I_{2}^{r}$, and forward bias, $I_{2}^{f}$, plotted against frequency of the applied bias are shown in Fig. 2. From its dc value of $\sim 8.5 \%$ at low frequency the value of $I_{2}^{r}$ is seen to rise to a maximum of $\sim 13 \%$ around $0.4 \mathrm{~Hz}$ from which it reduces at high frequencies to the value of $5 \%$. This latter value characterizes the system with a low residual $E L 2^{+}$charge layer behind the metal-semiconductor interface, ${ }^{5,8}$ and since a similarly low value is observed for $I_{2}^{f}$ at all frequencies it is concluded that trap filling in forward bias $\left(E L 2^{+}\right.$neutralization) is fast and essentially complete. In the same context, it is also noted that the dielectric relaxation time $(\varepsilon \rho)$ of our bulk SI-GaAs is $\sim 10^{-4} \mathrm{~s}$ and thus at the relatively low $(<\mathrm{kHz})$ frequencies of the present study there is no significant lag between the application of the bias and the onset of trap filling. 


\section{THEORY}

The observed frequency dependence of $I_{2}^{r}$ may be understood by first considering over one period of the applied bias, the spatial and temporal variations of the electric field, as sensed by the positron and that are produced by the $E L 2^{+}$ space charge in the sample. A neutral deep donor emits electrons to the conduction band at a rate per center given by

$$
e_{n}=N_{c} \sigma v \exp \left[-\left(E_{C}-E_{r}\right) / k_{B} T\right]
$$

while when ionized it captures electrons at a rate per center of

$$
c_{n}=n \sigma v,
$$

where $N_{C}$ is the conduction-band effective density of states, $\sigma$ is the thermal electron capture cross section, $v$ is the mean thermal electron velocity, $E_{C}-E_{T}$ is the energy difference between the deep level and the conduction band, and $n$ is the density of electrons in the conduction band. ${ }^{9}$ The density of ionized donors $N_{+}$is given by the rate equation. ${ }^{9}$

$$
\frac{d N_{+}}{d t}=e_{n}\left(N_{T}-N_{+}\right)-c_{n} N_{+},
$$

in which $N_{T}$ is the donor concentration and $c_{n}$ is that value pertaining to the spatial position being considered. As noted in Ref. $8, c_{n}$ approaches zero in reverse bias because $n$ approaches zero across the sample when carrier entry into the conduction band is blocked by way of the Schottky-like $\mathrm{Au} / \mathrm{GaAs}$ junction. Solving (3) in the reverse-biased half period $(0<t<\tau)$ the ionized donor concentration $N_{+}^{r}$ is found to be of the form

$$
N_{+}^{r}=N_{T}\left(1-A_{\tau} e^{-e_{n} t}\right),
$$

while in the forward-biased half period $(\tau<\mathrm{t}<2 \tau)$, when $n$ takes on the thermal equilibrium value as carriers move in from the bulk, the value of ionized donor concentration $\mathrm{N}_{+}^{f}$ is found to be

$$
N_{+}^{f}=N_{T} \beta+N_{T}\left[\left(1-A_{\tau} e^{-e_{n} \tau}\right)-\beta\right] e^{-e_{n} \beta^{-1}(t-\tau)},
$$

where $A_{\tau}=(1-\beta) \exp \left(-e_{n} \tau / 2\right) \sinh \left\{\left(e_{n}+c_{n}\right) \tau / 2\right\} / \sinh \left\{\left(2 e_{n}\right.\right.$ $\left.\left.+c_{n}\right) \tau / 2\right\}$ in both (4) and (5) and $\beta=e_{n} /\left(e_{n}+c_{n}\right)$.

To describe the spatial and temporal variations in the electric field, it is noted that under the "depletion approximation', 10 there are three discrete regions in the semiconductor. These are as shown in Fig. 3, and are, respectively, designated as region $1 A\left(0<x<W_{0}\right)$, which is always fully ionized through the natural band bending of the contact, region $1 B\left[W_{0}<x<W(t)\right]$ where ionization occurs as given by Eqs. (4) and (5), and region $1 C(\mathrm{x}>\mathrm{W}(\mathrm{t}))$ the electrically neutral bulk in which the ionized donor concentration is compensated by a residual ionized acceptor charge, $N_{\mathrm{A}}$ $\left(=N_{T} \beta\right)$. The mean electric fields in regions $1 A$ and $1 B$, obtained upon spatial averaging the electric fields within the sample of permittivity $\varepsilon$ and charge density $\rho=e\left(N_{+}-N_{A}\right)$, are

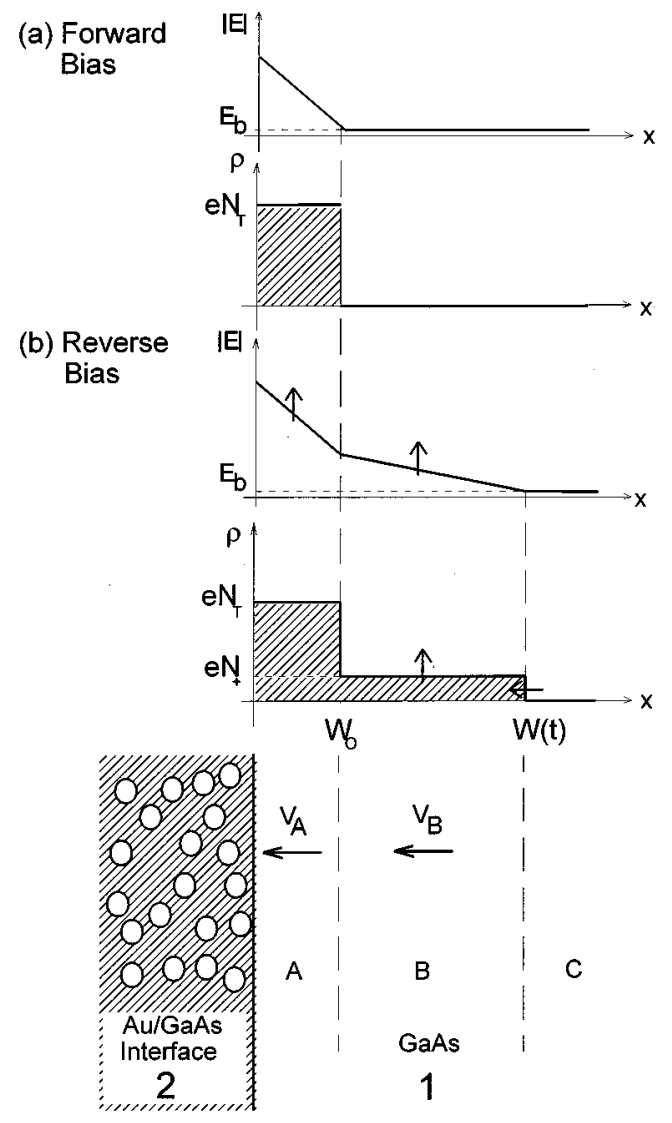

FIG. 3. A schematic diagram of the electric-field and chargedensity profiles in the SI-GaAs assumed in our model for (a) the reverse-biased and (b) the forward-biased $\mathrm{Au} / \mathrm{GaAs}$ junction. The lower diagram pictures the interface and electric-field regions and the open circles in the $\mathrm{Au} / \mathrm{GaAs}$ interface region 2 (annihilation rate $\lambda_{2}$ ) representing positron trapping microvoids. $v_{A}$ and $v_{B}$ are the positron drift velocities in the subregions $1 A$ and $1 B$, respectively, of the GaAs (annihilation rate $\lambda_{1}$ ).

$$
\begin{aligned}
\left\langle E_{1 A}(t)\right\rangle= & \frac{e N_{T}(1-\beta) W_{0}}{2 \varepsilon} \\
& +\frac{e\left[N_{+}(t)-N_{T} \beta\right]\left[W(t)-W_{0}\right]}{\varepsilon}+E_{b}, \\
\left\langle E_{1 B}(t)\right\rangle= & \frac{e\left[N_{+}(t)-N_{T} \beta\right]\left[W(t)-W_{0}\right]}{2 \varepsilon}+E_{b},
\end{aligned}
$$

in which $E_{b}=I(t) R_{b} / d$ is the electric field in region $1 C$, $I(t), R_{b}$, and $d$ being, respectively, the reverse current, the bulk resistance, and thickness of the sample, and

$$
W(t)=\left(\frac{2 \varepsilon}{e N_{+}(t)}\left\{V-I(t) R_{b}+\frac{N_{+}(t)}{N_{T}} \phi_{\mathrm{bi}}\right\}\right)^{1 / 2}
$$

is the depletion width which reduces to $W_{0}=\sqrt{2 \varepsilon \phi_{b i} / e N_{T}}$ when in forward bias $V-I R_{b} \cong 0, \phi_{\mathrm{bi}}$ is the built-in potential (band-bending) of the contact.

To describe positron drift to the interface an implantation profile of the form $P(x)=\alpha \exp (-\alpha x)$ is assumed, $\alpha^{-1}$ be- 
ing the mean implantation depth., ${ }^{5,8,11}$ The subsequent electric-field drift in regions $1 A$ and $1 B$ is approximated as uniform with mean drift velocities $v_{A}$ and $v_{B}$. Drift in region $1 C$ is ignored as the positron current from $1 C$ to $1 B$ is negligible at all field strengths $E_{b}$ encountered $(<500$ $\mathrm{V} \mathrm{cm}{ }^{-1}$ ). Taking the annihilation rates for drifting and microvoid trapped positrons as $\lambda_{1}$ and $\lambda_{2}$, respectively, and solving the rate equation for the number of positrons in the interface state $n_{2}(t)$ (Ref. 8) with $n_{2}(0)=0$ and an incoming positron current,

$$
\begin{gathered}
j(t)=N \alpha v_{A} e^{-\left(\alpha v_{A}+\lambda_{1}\right) t}, \quad 0<t<W_{0} / v_{A} \\
=N \alpha v_{B} e^{-\left(\alpha v_{B}+\lambda_{1}\right)-\alpha W_{0}\left[1-\left(v_{B} / v_{A}\right)\right]}, \\
W_{0} / v_{A}<t<W_{0} / v_{A}+\left[W(t)-W_{0}\right] / v_{B},
\end{gathered}
$$

an $\exp \left(-\lambda_{2} t\right)$ spectral component is found of intensity

$$
\begin{aligned}
i_{2}(t)= & \frac{\alpha v_{A}}{\alpha v_{A}+\Delta \lambda}\left\{1-e^{-\alpha W_{0}\left[1+\left(\lambda_{1} / \alpha v_{A}\right)\right]}\right. \\
& \times\left(1-\frac{\left(\alpha v_{A}+\Delta \lambda\right)}{\left(\alpha v_{B}+\Delta \lambda\right)} \frac{v_{B}}{v_{A}}\right. \\
& \left.\left.\times\left[1-e^{-\alpha\left(W(t)-W_{0}\right)\left[1+\left(\lambda_{1} / \alpha v_{B}\right)\right]}\right]\right)\right\},
\end{aligned}
$$

where $\Delta \lambda$ is the difference between bulk and interface annihilation rates $\lambda_{1}$ and $\lambda_{2} \cdot{ }^{12}$

\section{RESULTS AND DISCUSSION}

Equation (9) gives the instantaneous value of $i_{2}(t)$ and must be averaged either over the reverse half period to give $I_{2}^{r}$ or over the trap filling half period to give $I_{2}^{f}$. This has been done numerically using Eqs. (1), (2), and (4)-(7) together with the Bardeen-Schockley formula with longitudinal velocity of sound $4.8 \times 10^{5} \mathrm{~cm} \mathrm{~s}^{-1}$ and a low-field positron mobility of $45 \mathrm{~cm}^{2} \mathrm{~V}^{-1} \mathrm{~s}^{-1}$ for calculating the drift velocities $v_{\mathrm{A}}$ and $v_{B}$ from the mean fields of Eq. (6). ${ }^{5} \mathrm{In}$ application of Eqs. (1) and (2) we have as in Ref. 5 taken $v=4.5 \times 10^{7} \mathrm{~cm} \mathrm{~s}^{-1}$ and $N_{C}=4.7 \times 10^{17} \mathrm{~cm} \mathrm{~s}^{-1}$, while for Eqs. (4) and (5) $N_{T}$ has been taken as the quoted EL2 concentration of $1.5 \times 10^{16} \mathrm{~cm}^{-3}$. In computing Eqs. (6) and (7) we have taken $R_{b}=5 \times 10^{7} \Omega, d=0.05 \mathrm{~cm}$, and $\varepsilon=12.4 \varepsilon_{0} .5$ The dc current of $4 \times 10^{-6} \mathrm{~A}$ at the reverse bias $V=115 \mathrm{~V}$ was taken for $I(t)$ (transient currents from $E L 2^{+}$charging being always less than $1 \%$ of this de value). In computing $i_{2}(t)$ from Eq. (9) we have taken $\alpha=(50 \mu \mathrm{m})^{-1},{ }^{12} \lambda_{1}=(230$ $\mathrm{ps})^{-1,3}$ and $\lambda_{2}=(410 \mathrm{ps})^{-1}$. Since $A_{\tau}$, and hence $N_{+}^{r}$ through Eq. (4) depends just on the emission rate $e_{n}$ in the fast capture regime $\left(c_{n} \gg e_{n}\right), I_{2}^{r}$ is only $e_{n}$ and not $c_{n}$ dependent. A visual fitting (curve shown in Fig. 2) of the $I_{2}^{r}$ data gives a value for $e_{n}$ of $0.114 \pm 0.002 \mathrm{~s}^{-1}$. Apart from the lowest frequencies (below $\sim 20 \mathrm{mHz}$ ) the fit to the $I_{2}^{r}$ peak is in remarkably good agreement with experiment, showing the essential correctness of our model.

It is found that the position of the $I_{2}^{r}$ peak is sensitive to $E_{C^{-}} E_{T}$ and fairly insensitive to other parameters. This sensitivity arises from the strong exponential dependence of the electron emission rate $e_{n}$ on $E_{C^{-}} E_{T}$, as seen from Eq. (1), from which small changes in $E_{C^{-}} E_{T}$ cause large changes in $e_{n}$ if the temperature is held constant. Since $e_{n}$ depends on both $\sigma$ and $E_{C^{-}} E_{T}$ [Eq. (1)], neither of these quantities can be deduced independently of each other. Electron capture cross sections for EL2 are, however, generally accepted to be in the range of $1-3 \times 10^{-13} \mathrm{~cm}^{-2}$ (Refs. 13, 17-19) for doped materials. Adopting a value in this range for the present semi-insulating GaAs introduces an uncertainty of $\pm 0.02 \mathrm{eV}$ in the fitted value of $E_{C}-E_{T}$. This, however, is not the only systematic error on the trap energy. Uncertainty of the model parameters $\mu\left( \pm 15 \mathrm{~cm}^{2} \mathrm{~V}^{-1} \mathrm{~s}^{-1}\right)$ and $N_{T}$ $\left( \pm 5 \times 10^{15} \mathrm{~cm}^{-3}\right)$ introduces, respectively, additional uncertainties of $\pm 0.02 \mathrm{eV}$ in the trap energy. Changing the implantation parameter $\alpha$, however, over the relatively large range from $(40 \mu \mathrm{m})^{-1}$ to $(60 \mu \mathrm{m})^{-1}$ produces no perceptible change in the modeled peak position. We thus quote for $E_{C^{-}} E_{T}$ a fitted value of $0.80 \pm 0.01 \pm 0.05 \mathrm{eV}$, where the first error is statistical and the second systematic. The latter error is adopted as being reasonable since it lies between the extremes of perfect parameter correlation and total independence.

The value of $E_{C^{-}} E_{T}$ for $E L 2$ found in the literature has a wide range of quoted values ranging from 0.718 to $0.93 \mathrm{eV}$ with the typical quoted errors being less than $0.01 \mathrm{eV} .{ }^{13,14}$ However, for well characterized, non-semi-insulating material a value for $E_{C^{-}} E_{T}$ of between 0.81 and $0.83 \mathrm{eV}$ is most widely quoted. ${ }^{15-17}$ Such values are in good agreement with our present and previous work ${ }^{5}$ on semi-insulating GaAs, indicating that the doping of the material does not have a marked influence on the structure of the EL2 defect.

The fitted curve for the forward-biased intensity $I_{2}^{f}$, with $\phi_{\mathrm{bi}}=6 \mathrm{eV}$, is also shown in Fig. 2. Again a reasonable representation of the data is obtained. In contrast to the $I_{2}^{f}$ data, $I_{2}^{f}$ does depend slightly on the capture rate $c_{n}$ and hence capture cross section $\sigma$. This is because of the less effective $E L 2^{+}$neutralization that occurs at high frequencies where carrier capture occurs for only half the available time. Unfortunately, our $I_{2}^{f}$ data are of insufficient accuracy to allow any precise determination of $\sigma$, but the observed rise of $\sim 1 \%$ in intensity at frequencies above $\sim 100 \mathrm{~Hz}$ is quite consistent with the above-mentioned range of cross sections for the EL2 defect.

The unrealistically large fitted natural band-bending value of $\phi_{\mathrm{bi}}=6 \pm 2 \mathrm{eV}$ deserves further comment. This parameter is dominant in controlling the value of $I_{2}^{f}$ and the highfrequency value of $I_{2}^{r}$. Thus an error in compensating for source annihilations could account for the large value. On the other hand, as considered in Ref. 8, it is likely that some of the contribution to $I_{2}^{f}$ results from a near surface super exponential form for the implantation profile. Our model, assuming a perfect exponential implantation of thermalized positrons over the whole sample, underestimates the fraction of positrons stopping within the natural depletion width $\left(W_{0}\right)$. In addition, the breakdown of the depletion approximation, assumed in the above modeling, at the metal-semiinsulator junction could also be playing a role. ${ }^{8}$ The only parameter in our model that can compensate for such errors is $\phi_{\mathrm{bi}}$ which must be set at unrealistically large values in order to mimic a larger depletion region for capturing positrons. The same arguments also explain why the lowfrequency reverse-bias data is high compared to the modeled 
curve, for at low frequencies the depletion zone is narrow and positrons are preferentially collected from the near surface region.

The $I_{2}^{r}$ peak results from two competing processes. In the first place the mean electric field $E$ experienced by positrons in the depletion region increases steadily with time in reverse bias, which leads to $I_{2}^{r}$ monotonically increasing (as $\sim \alpha \mu_{+} E /\left\{\alpha \mu_{+} E+\Delta \lambda\right\}$ ) with bias period. However, as the electric field is increasing, the width of the depletion region is decreasing and this leads to a lesser fraction $\left[\sim\left(1-e^{-\alpha W}\right)\right]$ of the implanted positrons being within the strong field of the depletion region as time elapses. There is thus some optimal elapsed time, after the onset of reverse bias, for which the positron current to the interface is maximized, giving rise to some optimal frequency $(\sim 0.4 \mathrm{~Hz}$ at $295 \mathrm{~K})$ for maximizing $I_{2}^{r} .^{21}$

\section{CONCLUSION}

In summary, the present study has demonstrated that the type of information available using a conventional DLTS technique, such as trap energies and cross sections, can be obtained to a reasonable degree of accuracy by monitoring the transient electric fields in the depletion region of a semiconductor by way of positron drift to an interface. It also indicates that such positron studies may in some instances offer advantages over conventional DLTS, such as, for example, not necessarily requiring temperature variation for the determination of trap energy (although without doubt variation of temperature and a subsequent Arrhenius plot would lead to a more accurate value being obtained). Moreover, the fact that the positron can respond to electric-field transients occurring down to the nanosecond time range (because of its short lifetime $\sim 0.2 \mathrm{~ns}$ in semiconductors) could also lend an advantage over capacitance transient measurements, since the latter cannot be effectively measured at submillisecond times. Such fast measurement of electric-field transients would mean that trap levels occurring much closer to the conduction and valence bands could become accessible at liquid-nitrogen temperatures and above. For such measurements on doped semiconductors the use of a low-energy positron beam for implanting positrons into narrow depletion regions would be necessary and would greatly enhance the sensitivity of this type of measurement.

\section{ACKNOWLEDGMENT}

Helpful discussions with C. V. Reddy concerning the DLTS technique are gratefully acknowledged.
${ }^{1}$ K. Saarinen, P. Hautojärvi, P. Lanki, and C. Corbel, Phys. Rev. B 44, 10585 (1991).

${ }^{2}$ C. Le Berre, C. Corbel, R. Mih, M. R. Brozel, S. Tüzemen, S. Kuisma, K. Saarinen, P. Hautojärvi, and R. Fornari, Appl. Phys. Lett. 66, 2534 (1995).

${ }^{3}$ C. Corbel, F. Pierre, K. Saarinen, P. Hautojärvi, and P. Moser, Phys. Rev. B 45, 3386 (1992).

${ }^{4}$ R. Krause, K. Saarinen, P. Hautojärvi, A. Polity, G. Gärtner, and C. Corbel, Phys. Rev. Lett. 65, 3329 (1990).

${ }^{5}$ H. L. Au, C. C. Ling, B. K. Panda, T. C. Lee, C. D. Beling, and S. Fung, Phys. Rev. Lett. 73, 2732 (1994).

${ }^{6}$ For general review of DLTS see, e.g., G. L. Miller, D. V. Lang, and L. C. Kimberling, Annu. Rev. Mater. Sci. 1977, 377.

${ }^{7}$ H. L. Au, P Asoka-Kumar, C. D. Beling, S. Fung, and K. G. Lynn, Phys. Status Solidi B 178, K11 (1993).

${ }^{8}$ Y. Y. Shan, H. L. Au, C. C. Ling, T. C. Lee, B. K. Panda, S. Fung, C. D. Beling, Y. Y. Wang, and H. M. Weng, Appl. Phys. A 59, 259 (1994).

${ }^{9}$ See, for example, M. Lanoo, and J. Bourgoin, Point Defects in Semiconductors II (Springer-Verlag, Berlin, 1983), p. 157.

${ }^{10}$ E. H. Rhoderick and R. H. Williams, Metal-Semiconductor Contacts, 2nd. ed. (Clarendon, Oxford, 1988).

${ }^{11}$ W. Brandt and R. Paulin, Phys. Rev. B 15, 2511 (1977).
${ }^{12}$ See AIP Document No. PAPS PRBMDO-55-7624-3 for 3 pages of a calculation of the positron lifetime spectral term. Order by PAPS number and journal reference from American Institute of Physics, Physics Auxiliary Publications Service, Carolyn Gehlbach, 500 Sunnyside Boulevard, Woodbury, New York 117972999. Fax: 516-576-2223, e-mail: paps@aip.org. The price is $\$ 1.50$ for each microfiche (98 pages) or $\$ 5.00$ for photocopies of up to 30 pages, and $\$ 0.15$ for each additional page over 30 pages. Airmail additional. Make checks payable to the American Institute of Physics.

${ }^{13}$ D. C. Look, Electrical Characterization of GaAs Materials and Devices (Wiley, New York, 1989), p. 202.

${ }^{14}$ Ming-Fu Li, Modern Semiconductor Quantum Physics (World Scientific, Singapore, 1994), p. 332.

${ }^{15}$ J. Lagowski, H. C. Gatos, J. M. Parsley, K. Wada, M. Kaminska, and W. Walukiewicz, Appl. Phys. Lett. 40, 342 (1982).

${ }^{16}$ M. Kaminska, M. Skowronski, and W. Kuszko, Phys. Rev. Lett. 55, 2204 (1985).

${ }^{17}$ F. D. Auret, A. W. R. Leitch, and J. S. Vermaak, J. Appl. Phys. 59, 158 (1986).

${ }^{18}$ G. M. Martin, A. Mitonneau, and A. Mircea, Electron. Lett. 13, 191 (1977).

${ }^{19}$ R. Y. Dejule, M. A. Haase, D. S. Ruby, and G. E. Stillman, Solid-State Electron. 28, 639 (1985). 